\title{
Urgences
}

\section{Un homme et son péché sous la plume des correcteurs}

\section{Yvette Francoli et Antoine Sirois}

Numéro 24, juillet 1989

Le manuscrit sous l'angle

URI : https://id.erudit.org/iderudit/025527ar

DOI : https://doi.org/10.7202/025527ar

Aller au sommaire du numéro

Éditeur(s)

Urgences

ISSN

0226-9554 (imprimé)

1927-3924 (numérique)

Découvrir la revue

Citer cet article

Francoli, Y. \& Sirois, A. (1989). Un homme et son péché sous la plume des correcteurs. Urgences, (24), 7-15. https://doi.org/10.7202/025527ar d'utilisation que vous pouvez consulter en ligne.

https://apropos.erudit.org/fr/usagers/politique-dutilisation/ 


\section{Un homme et son péché sous la plume des correcteurs \\ Yvette Francoli et Antoine Sirois, Université de Sherbrooke.}

Un homme et son péché est un court roman de 200 pages seulement que son auteur a peaufiné avec un soin jaloux, un peu comme le peintre apporte retouches sur retoúches à sa toile sans pouvoir la déclarer achevée. C'est sans doute pour ne plus être tenté d'y revenir que Grignon a fait de l'édition de 1985 une "édition définitive". En ce qui nous concerne, nous avons travaillé à partir d'une version dactylographiée, corrigée de la main de Grignon, le premier jet manuscrit ayant, semble-t-il, disparu. Mais entre la première édition de 1933 et ce «tapuscrit" (comme il a été convenu de l'appeler), il y a de nombreux remaniements et variantes de texte, ce qui tend à prouver que les corrections ont bien été faites sur les épreuves ainsi que Grignon l'a affirmé. Parmi les trois correcteurs qui ont aidé Grignon à amender les versions successives d'Un homme et son péché, il y a d'abord eu son éditeur, Albert Pelletier (Éditions du Totem) avec lequel il passa de nombreuses fins de semaine à corriger les épreuves de l'édition 1933: "en ce temps-là, beaucoup plus qu'aujourd'hui, corriger des épreuves, c'était écrire deux fois". "C'est, a-t-il confié dans ses écrits inédits, une sensation indéfinissable de bien-être. C'est une joie que l'on fait durer comme à plaisir". " II ne reste malheureusement aucune trace de ces corrections, les épreuves ayant été probablement détruites dans l'incendie de la maison des Pelletier. Mais ainsi que devait le reconnaître Grignon lui-même, Pelletier avait eu le grand mérite de lui "laisser ses canadianismes, ses archaïsmes, ses provincialismes et, pour tout dire, un patois qu'il estimait savoureux".2

Après Pelletier, Grignon s'adressa à deux autres correcteurs chevronnés en vue de "l'édition définitive" de 1935 qu'il comptait présenter - et présenta - au Prix David: LouisPhilippe Geoffrion, auteur de nombreux ouvrages sur la langue

1 Claude-Henri Grignon, «Le pamphlétaire maudit ", 1'o partie, f.8 (fonds privé).

2 lbid. 
française (envers lequel il a avoué sa dette dans les nombreuses conférences qu'il consacra à Un homme et son péché) et Louvigny de Montigny, homme de lettres, critique et traducteur parlementaire qu'il devait par la suite vilipender dans ses Pamphlets. Nous possédons les exemplaires corrigés de leur main, ce qui nous a permis d'authentifier les variantes entre les éditions de 1933 et de 1935. Il existe également un exemplaire sur lequel Grignon a recopié avec une application d'écolier toutes les corrections et suggestions proposées par ses correcteurs, y ajoutant ici et là quelques-unes de son cru.

Les corrections de L.-P. Geoffrion se bornent à quelques détails de style, de ponctuation, d'orthographe ("chariot" pour "charriot", "flanelette" pour "flanalette", "rechausser" pour "renchausser", etc.), de chiffres à transcrire en toutes lettres, de réécriture de certains mots du terroir, comme "icitte" pour "icit"», ou encore "p'en tout». pour «pantoute" (propositions qui ne seront pas retenues par Grignon).

II suggère le remaniement de certains passages où l'image lui a paru obscure ou recherchée. II rejette quelques canadianismes en faveur d'équivalents plus académiques: "économiser" pour "sauver" ou "ménager", "seau" pour "chaudière", "berceuse" pour "berçante", "pierres" pour "roches". Mais là s'arrête son intervention.

Par contre, Louvigny de Montigny s'est montré d'une rigueur toute académique. "Puriste" intransigeant, il sabre le texte à la recherche du mot juste, concis. II rejette les banalités, les mièvreries, n'hésitant pas au besoin à donner en marge du texte de nombreux commentaires tant sur le style que sur le choix des mots ou des métaphores, la psychologie des personnages, l'agencement des épisodes. II conseillera par exemple, pour l'intelligence du récit, de justifier les causes de l'incendie, c'està-dire de signaler en quelque sorte du doigt le présumé coupable. Il eût suffi selon lui de laisser rôdailler "l'Homme aux cheveux roux" autour de la maison, pour lui donner le temps d'y mettre le feu, et le lecteur eût compris que c'était lui qui venait se venger. Grignon préférera finalement une fin plus ambiguë. Le lecteur a en effet le choix entre la justice divine, l'acte de vengeance, ou tout bonnement l'accident. Se prévalant de son droit de correcteur, Louvigny se permet des remarques qui laissent parfois percer son impatience ou son ironie. Par exemple, l'image "son corps, prenait la forme d'une chose inerte", soulève chez lui la question suivante: "Est-ce "la forme" 
qui distingue une chose inerte d'une chose qui ne l'est pas?" Et la réflexion de Donalda, constatant le retour tardif de son mari: "Est-ce que je rêve, se demanda-t-elle?" en appelle une autre de sa part: "L'arrivée de la voiture de l'avare bien qu'en retard, avait-elle de quoi donner à rêver à Donalda?" En marge de la description d'une table "aux pattes énormes représentant des têtes de lion», Montigny écrit: “Des pattes ou des pieds qui représentent des têtes de lion!" Face aux "effluves qui poussent l'homme aux combats contre la matière et vers les plus beaux désirs", il s'exclame avec raison: "Qu'est-ce que cela peut bien vouloir dire?" À la suite de "Séraphin tira sur le corps en élevant la tête et les genoux", il ajoute avec humeur: "Analysez cette manœuvre si vous pouvez!» Plus Montigny avance dans son travail de correction, plus il souligne ou supprime des mots, des corps de phrases, des paragraphes entiers, plus il parsème le texte de points d'interrogation ou d'exclamation, de remarques parfois acerbes ou agacées, ce que Grignon ne lui pardonnera jamais, bien qu'il ait suivi la plupart de ses suggestions de style: «Mon livre ne vaut rien et je le sais, c'est l'opinion à la barbiche de Louvigny de Montigny et ce Français d'Anglais ne peut pas se tromper".3

C'est vraisemblablement en pensant à lui que Grignon dira lors d'un dîner-causerie: "La langue française dans toute sa correction repose sur l'usage. C'est l'usage qui fait la langue. Pas les grammairiens ni les professeurs, encore moins les puristes. Ces gens-là ne vivent que pour étriquer la langue, la rendre informe, conventionnelle, incolore et précieuse". 4

Louvigny de Montigny lui a fait l'affront de rejeter systématiquement tout canadianisme, toute trace de vocabulaire paysan. II ne veut rien entendre, par exemple, du mot "pesat" qu'il qualifie de "beau mot paysan à employer quelque part, mais pas ici". II rejette pour la même raison l'expression "quand je l'ai mariée": "Un paysan peut employer "marier" dans ce sens, mais non l'auteur", professe-t-il en marge de l'ouvrage. II craint, par ailleurs, que le lecteur français ne puisse comprendre le terme "ouaguine". II propose "fermé" pour "barré à clef", "le

3 Claude-Henri-Grignon, “Son Excellence est bien bonne», Les pamphlets de Valdombre, 1ro année, no 4, 1 or mars 1937, p. 155.

4 Idem, Dîner-causerie, Chambre de commerce de Sainte-Adèle, 29 novembre 1939 (fonds privé). 
sein" pour la "mamelle", "épargner" pour "sauver, "mon père" pour "poupa", "le cercueil» pour "la tombe", etc. Si Grignon ignore de telles corrections, par contre il adopte aussitôt l'expression archaïque "je tirerai les vaches", au lieu de "je trairai" que lui propose assez paradoxalement Montigny. Car c'est par souci de "faire vrai" qu'il emploie la langue familière et aussi pour se faire comprendre du grand public:

Je m'adresse au peuple. C'est écrit le plus simplement du monde. Je ne sais pas s'il a fallu un vocabulaire de 500 mots. Sur un roman de 200 pages, il y a seulement 14 canadianismes qui sont tirés de l'anglais. Le mot asmatten, c'est un mot savoureux, «smart», généreux, habile... alors moi je $n$ ' dis pas «smart", j' dis «smatte»... «Quelle fille smatte, pensa Séraphin $m .5$

Au sujet du mot "ouaguine" par exemple, qui n'existe dans aucun lexique de langue canadienne, il avoue qu'il l'a tout simplement fabriqué: "Si j'avais écrit "un chariot de ferme", y a pas un habitant qui aurait acheté mon livre". 6 On sent en effet qu'en écrivant son récit, Grignon a prononcé à haute voix les répliques de ses personnages pour rendre avec le plus d'exactitude possible sa vision du monde paysan. Pour lui, ala pierre de touche du roman, c'est le dialogue". ${ }^{7} \mathrm{Ne}$ suit-il pas l'exemple même de Molière, qu'il connaît "presque par cœur».

Si, avec Un homme et son péché, Grignon visait surtout le lecteur canadien-français, c'est parce qu'il ne se faisait aucune illusion sur ses chances d'être lu par un public français:

II est faux que nous puissions vendre nos œuvres en France. Jamais un écrivain français (je parle d'un écrivain digne de ce nom) ne s'abrutira à lire nos sentimentaleries. Et si, par miracle, nous produisons un chef-d'œuvre, je doute qu'un écrivain ou un critique français se charge de le lancer à Paris.

5 Claude-Henri Grignon, Précisions sur Un homme et son péché, Montréal, Éditions du Vieux Chêne, 1936, p. 106.

6 Émission radiophonique avec Albert Le Grand et Gilles Marcotte, Aujourd'hui, 18 mars 1965.

7 Causerie inédite donnée par Grignon au Club Saint-Laurent Kiwanis, à Montréal, le 2 mars 1949 (fonds privé). «Molière, avait-il commencé, est le plus bel exemple de la perfection du langage au théâtre, c'est-à-dire du langage de ses personnages [...] Molière se fit applaudir et comprendre par le peuple à cause du langage parlé, et le grand Molière entrait dans la gloire». 
Pour la simple raison que les littérateurs français en ont assez de vendre leurs propres ouvrages. ${ }^{8}$

Pour sa part, ses tentatives auprès de quelques «écrivains qui comptent parmi les plus grands en France", pour introduire Un homme et son péché sur le marché du livre français, s'étant soldées par un cuisant échec, force lui fut de reconnaître "l'indifférence que professent les littérateurs français à l'endroit des bons Canayens, littérateurs en souyés de beu!»

Convaincu, cependant, que l'imitation servile des modèles français, tant au niveau de la langue qu'à celui des idées, est à la source même de la médiocrité de la littérature canadienne, il écrivai:

Nous avons une langue mi-française, mi-canadienne, apprise au foyer sur les genoux du peuple. C'est celle qui devra servir au littérateur de chez nous. Nous avons besoin d'un dictionnaire canadien fait intelligemment non pas par des grammairiens, mais par des poètes du terroir et par les écrivains exotistes qui possèdent à la perfection l'intelligence des mots, laquelle donnera naissance à des associations d'idées multiples. Restons Canadiens. Écrivons le mot canadien sans l'affubler de guillemets avec le plus grand naturel du monde. Les Français, les étrangers qui nous liront se donneront la peine de chercher la signification de ce mot. $\mathrm{Ne}$ craignons pas les canadianismes. Ils sont beaux, pleins de couleurs et de sons. ${ }^{9}$

On ne peut, en effet, l'accuser d'avoir abusé des guillemets dans son propre texte. Nous n'en avons dénombré que trois: "belle érable franche", "veillée au corps" et draveurs "d'en haut". II reprochera à Félix-Antoine Savard, dont il a lu pourtant avec enthousiasme le Menaud, maître-draveur, d'avoir encadré de guillemets malheureux "ces beaux mots du pays de Québec", tels que "drave", "draveur", "bleuetière", "abatis", "corps-morts", "portage", "boucane", "poudrerie", sans lesquels Menaud ne serait rien. Il vénère les vieux mots du terroir que l'on retrouve encore sous la plume des écrivains régionalistes français: “icite», "veilleux", "tissure», «talle»,

8 Claude-Henri Grignon, «Faits et gestes - Son Excellence est bien bonne', Les pamphlets de Valdombre, $1^{\text {re }}$ année, no 4, $1^{\text {er mars } 1937,}$ p. 158.

9 Idem, Ombres et clameurs. Regard sur la littérature canadienne, Montréal, Éditions Albert Lévesque, 1933, p. 189. 
"seillon", "bourdignond", mais que "des Français à la gueule fine et davantage l'Académie ont commis la sottise de laisser choir, un à un". ${ }^{10}$ Selon lui, l'écrivain canadien se doit d'employer couramment cette langue vivante car c'est le seul moyen de créer "une littérature nationale". 11

Cependant, pour les critiques soucieux de "purisme", les "échantillons de langage canadien" qui émaillent le texte d'Un homme et son péché font l'effet "d'oublis regrettables". Pour les autres, Grignon fait figure d'innovateur. II ose ce que personne n'avait osé, c'est-à-dire introduire dans des phrases toutes françaises des expressions canadiennes, voire "argotiques", pour reprendre le terme de Louis Dantin. "Comment te sens-tu à matin", "Quoi c'est qu'elle a", "Qui c'est qui pourrait me voler", "Je m'en vas-t-y aller", "Ça fait-y longtemps qui est d'même". II ne reculera même pas devant les fautes d'orthographe que pourrait faire l'habitant quasi analphabète, comme par exemple: “Ugnon d'prières" que Louvigny de Montigny ne tolérera pas: "Inutile de phonographier le langage des paysans et nulle part ailleurs". C'est à lui vraisemblablement que nous devons la suppression des élisions et de l'orthographe phonétique des dialogues.

Bien que Grignon n'ait pas toujours suivi à la lettre les recommandations de ses correcteurs, il n'en a pas moins reconnu l'utilité d'un tel procédé et conseillé aux jeunes écrivains de ne jamais livrer un manuscrit à un imprimeur avant de l'avoir soumis à "deux ou trois esprits éclairés et libres":

Cette méthode très française, en outre de vous fournir des connaissances indispensables, vous apprendra qu'il est bien difficile de rencontrer deux juges qui puissent s'entendre sur telle ou telle question de langage. Personnellement, j'ai tiré profit de cette expérience. J'ai découvert aussi où se trouvent les envieux, les cœurs secs, les ennemis véritables et les amis. Tel critique éplucha mon livre au point qu'il n'y avait pas un mot de bon. Celui-là exagérait j'en suis certain. ${ }^{12}$

Ici, "l'ennemi véritable" est de toute évidence Louvigny de Montigny. L'ironie du sort fera que ce dernier se portera candidat

10 Idem, «Au pays de Québec, s'agirait-il d'un chef-d'œuvre?», Les pamphlets de Valdombre, $1^{10}$ année, no 9, 1 er août 1937, p. 392-393.

11 Ibid., p. 393.

12 Idem, Précisions sur Un homme et son péché, p. 80. 
au Prix David, la même année que Grignon, avec sa pièce de théâtre Les boules de neige, ce qui fera dire à Grignon amusé: "Je ne vous veux pas de mal, vous le savez, encore que le titre de mon récit ait fait fondre devant le Prix David, en 1935, vos Boules de neige. ${ }^{13}$ Précisons que ce persiflage fait suite à une "énormité" que Grignon a lue dans la thèse de doctorat de Louvigny, qui prétend qu'Un homme et son péché est "un tableau de la dépravation des mœurs dans nos campagnes canadiennes-françaises". 14

La réalité paysanne et villageoise est le seul guide de Grignon dans sa recherche du mot juste et de l'expression exacte et forte. II est le peintre de la vie rurale et tient à ce qu'on ne l'oublie pas: "Veuillez donc rappeler à tous vos lecteurs, écrit-il à Olivar Asselin, que le roman a été conçu et écrit à Sainte-Adèle, Comté de Terrebonne, patrie des tonitruants curé Labelle et Dr Wilfrid Grignon. ${ }^{15}$ C'est pour eux qu'il conserva, en dépit des suggestions de ses correcteurs et des critiques littéraires, ces "beaux mot du pays de Québec": "noirceur", "chaise berçante", "barrée à clé", "chaudière", "chanceuse", "malle", "pesat". Il fera toutefois quelques concessions aux

13 Idem, "Médecin, guéris-toi toi-même, Les pamphlets de Valdombre, $2^{\theta}$ année, no 4, mars 1938, p. 158-159. Les boules de neige, comédie suivie d'un sketch en un acte. Je vous aime avait été publiée en 1935. Alphonse Désilets prédisait que ces deux comédies feraient "leur chemin dans le domaine théâtral au Canada français", Le terroir, vol. 18, no 10, mars 1936, p. 15.

14 Voici, dans son intégralité, ce passage de la thèse de doctorat de L. de Montigny", "La revanche de Maria Chapdelaine", D.L. Montréal, 1939, tel que cité par Grignon: a Un homme et son péché n'est-il pas l'histoire d'un répugnant avare qui assassine sa jeune femme à force de privations, et qui s'entoure accessoirement de hideux lubriques parmi lesquels brille une petite prostituée à qui, pour bien la désigner comme telle, l'auteur inflige le vocable le plus clair en même temps que le plus grossier du vocabulaire? Ce roman de Claude-Henri Grignon est proprement un tableau de la dépravation des mœurs dans nos campagnes canadiennes-françaises». Grignon avoue mieux comprendre à la lecture de ces lignes la mise en garde d'Olivar Asselin, quelques mois avant sa mort: «Valdombre, faites attention à Louvigny de Montigny. C'est un prude qui a peur des mots et qui passe son temps à en traduire» (Les pamphlets de Valdombre, no 4, mars 1938, p. $153-163$.

15 Lettre de C.-H. Grignon à Olivar Asselin, 29 septembre 1935 (fonds privé). 
puristes en adoptant "sous" au lieu de "cents", "dollars" pour "piastres", "cigare" pour "Peg Top". II supprimera aussi de nombreux «ben" et consentira à ce que Séraphin "épouse" et non pas «marie" la petite Donalda Laloge. Si Grignon valorise l'emploi de la langue vernaculaire dans les scènes de la vie quotidienne, par contre dans les scènes de grande intensité dramatique, comme celle de l'agonie de Donalda, il redevient un écrivain classique. À part quelques faits phonétiques ("v'là", "ben", "à c't'heure", "m'man") et lexicaux ("patate») tous dans le dialogue (ici le délire de la mourante), son style est d'une rigueur toute académique.

On peut dire que sa tâche principale consiste à remplacer par une image simple l'image incohérente que le besoin d'une impression forte lui avait inspirée. Dans la première coulée, Grignon semble avoir craint de ne pas rendre assez suggestives les moindres nuances psychologiques, d'où l'emploi abusif d'images et de métaphores qu'il jugera par la suite pour le moins "cocasses" ou "bouffonnes", ce que Louvigny avait désavoué. Ainsi disparaissent "les bains fugaces de délices", "les bouffées du désir", "les aiguilles de l'impureté", "les labyrinthes toujours quelque peu inédits des billets à ordre", et le long passage consacré aux gambades des animaux qui formaient "un ballet impromptu à la gloire de la résurrection du sol sous les baisers de la lumière..."

L'allègement du style se manifeste également par la lutte contre l'envahissement des qualificatifs. Asselin, et plus tard Dantin, avaient dénoncé la surabondance adjective d'Un homme et son péche: "Avons-nous besoin de tant d'épithètes? [...], demandait Dantin, qu'on rende les actes mêmes éloquents, suggestifs: ils se qualifieront tout seuls". ${ }^{16} \mathrm{Et}$ d'ajouter: "Au lieu d'"elle se tordait, la pauvre Donalda, comme sur un lit de braise", il valait mieux écrire: "Elle se tordait comme sur un lit de braise", et nous eussions songé: "la pauvre Donalda!" "Grignon devait en tenir compte et non seulement supprimer "la pauvre Donalda", mais toutes les épithètes inutiles qui parsemaient le texte. II biffera par exemple: "les sapins noirs [silencieux et stö̈ques]" ou encore "le froid cinglant du nord, [horizontal, plein et enveloppant]". Il aura soin également de mettre un frein à la surabondance adverbiale. II

16 Louis Dantin, Gloses critiques, p. 131. 
supprimera par exemple: "La douleur disparaissait miraculeusement" ou encore: "Elle connaissait bien le mécanisme", "Le feu qui s'en allait lentement". II remplacera d'autre part tous les "tranquillement" du texte original par l'adverbe "doucement ". Il le regrettera plus tard:

Bernanos écrit toujours «tranquillement» pour adoucement", "paisiblement», «avec calme». Voilà qui en bouche un coin à certain critique envieux et ignare qui me reprochait des "tranquillement» dans mon roman, Un homme et son péché. Et quand on sait que Bernanos est un maître de la langue française, franchement et tranquillement, j'en tire gloire et orgueil. ${ }^{17}$

II n'est pas loin de désavouer son "flaubertisme", selon le mot d'Albert Pelletier qui, lors de la parution de l'édition définitive, avait raillé chez Grignon la manie de se "re-corriger". Ce sont peut-être les remarques de son premier correcteur qui lui avaient fait regretter son excès de zèle: "J'ai corrigé sérieusement Un homme et son péché, j'aurais peut-être mieux fait de corriger légèrement. Je me suis probablement trompé, qui ne se trompe pas? "18 Mais, à cette époque, Grignon doutait à la fois de son français et de ses qualités de romancier, d'où son besoin de justifier son œuvre dans cette longue conférence qu'il publiera sous le titre de Précisions sur Un homme et son péché, d'où également son initiative de recourir à des correcteurs chevronnés. N'avait-il pas déjà coutume de demander à Olivar Asselin de "biffer" et de "corriger" les articles qu'il lui envoyait pour publication: “J'ignore ma langue, qui peut se vanter de la connaître? „19

Nous conclurons pour notre part, à la suite de Rex Desmarchais, qu'avec Un homme et son péché, Claude-Henri Grignon "a donné la preuve qu'il écrivait une langue animée, savoureuse, pleine de couleur et de mouvement, une langue très honorable pour un écrivain des années trente".

17 Claude-Henri Grignon, "Les livres. Bernanos au presbytère», Les pamphlets de Valdombre, no 2, 1 or janvier 1937, p. 50.

18 aMoi, sociable», La renaissance, 7 décembre 1935, p. 5.

19 Lettre inédite de C.-H. Grignon à O. Asselin, 24 avril 1931. 